\title{
Stoma-Const - the technical aspects of stoma construction: study protocol for a randomised controlled trial
}

\author{
Adiela Correa Marinez' ${ }^{1}$ Sofia Erestam ${ }^{1,2}$, Eva Haglind ${ }^{1}$, Jan Ekelund ${ }^{1}$, Ulf Angerås', Jacob Rosenberg 3 , \\ Frederik Helgstrand ${ }^{4}$ and Eva Angenete ${ }^{1 *}$
}

\begin{abstract}
Background: The construction of a colostomy is a common procedure, but the evidence for the different parts of the construction of the colostomy is lacking. Parastomal hernia is a common complication of colostomy formation. The aim of this study is to standardise the colostomy formation and to compare three types of colostomy formation (one including a mesh) regarding the development of parastomal hernia.

Methods/Design: Stoma-Const is a Scandinavian randomised trial comparing three types of colostomy formation. The primary endpoint is parastomal herniation as shown by clinical examination or CT scan within one year. Secondary endpoints are re-admission rate, postoperative complications (classified according to Clavien-Dindo), stoma-related complications (registered in the case record form at stoma care nurse follow-up), total length of hospital stay during 12 months, health-related quality of life and health economic analysis as well as re-operation rate and mortality within 30 days and 12 months of primary surgery. Follow-up is scheduled at 4-6 weeks, and 6 and 12 months. Inclusion is set at 240 patients.

Discussion: Parastomal hernia is a common complication after colostomy formation. Several studies have been performed with the aim to reduce the rate of this complication. However, none are fully conclusive and data on quality of life and health economy are lacking. The aim of this study is to develop new standardised techniques for colostomy formation and evaluate this with patient reported outcomes as well as clinical and radiological assessment.
\end{abstract}

Trial registration: Clinicaltrials.gov, NCT01694238.2012-09-24.

Keywords: Parastomal hernia, Colostomy, Mesh, Prevention

\section{Background}

Colostomy formation has been a standard surgical procedure for more than 100 years. A well-functioning colostomy may not negatively affect the patient's quality of life (QoL) [1]; however, this can only be said if the stoma is well functioning and if complications are kept to a minimum. The complication rates after stoma formation are considerable, with figures of $21-70 \%[2,3]$, and studies have shown that adequate stoma height, type of stoma,

\footnotetext{
* Correspondence: eva.angenete@vgregion.se

'Department of Surgery, Institute of Clinical Sciences, SSORG - Scandinavian Surgical Outcomes Research Group, University of Gothenburg, Sahlgrenska University Hospital/Östra, 41685 Gothenburg, Sweden

Full list of author information is available at the end of the article
}

body mass index (BMI), emergency surgery and gender may be of importance in reducing the risk of complications in both the short- and long-term perspectives [4-6].

The surgical technique of stoma formation is only partly evidence based. There are few studies focused on technical details about stoma construction and their future impact on stoma function. One study has attempted to standardise the skin incision to two-thirds of the width of the bowel [7], although the actual impact of this on the functional outcome of the stoma function was not presented. In the surgical literature a cruciate incision in the fascia and extraction of the bowel through a hole sufficient in size is a standard description of the surgical technique [8]. In clinical practice the sufficient 
size of the hole has often been equal to 'two fingerswidth', which is a fairly inexact measurement.

Parastomal hernia is a long-term complication that is common; in the literature figures up to almost $50 \%$ have been reported $[9,10]$. There have been discussions regarding the placement of the stoma and effects on hernia incidence $[11,12]$; however, no studies have been sufficient in design or size to thoroughly answer the question. Attempts to reduce the rates of parastomal hernias have been made in the last few years with the placement of a mesh at the construction of the stoma [13-18]. However, these trials did not include studies of health-related QoL or health economic aspects. The placement of a prophylactic parastomal mesh has not been universally accepted, mainly due to a hesitance related to the possible risk of infection with a foreign body, but also as most studies were small in sample size [19]. Another suggestion for the basic construction of the stoma has been to make a circular instead of a cruciate incision in the fascia, but this has not been studied. It has been described in conjunction with the use of circular stapling devices, and no hernias were found, but the studies were small $[20,21]$. Thus, it is apparent that further studies are desired [17,22-24] and several randomised controlled trials are registered and ongoing.

The evaluation of parastomal hernias can be performed by clinical examination, CT scan or ultrasound $[14,15,25,26]$. All techniques have advantages and disadvantages, and the conclusion must be that evaluation of parastomal hernias may be difficult and must be standardised in a study.

The hypothesis to be tested in this study is that it is possible to obtain a lower incidence of parastomal hernia with a circular incision in the abdominal fascia or with reinforcement with a mesh instead of a cruciate incision in the abdominal fascia.

The study will provide a precise control compared to the two interventions with thorough measurements of the bowel and the trephine opening to improve the evaluation of the surgical technique. It will also enable an evaluation of QoL and health economy.

\section{Methods}

\section{Study objective}

The Stoma-Const trial is a randomised trial comparing three different surgical procedures for the construction of a colostomy.

\section{Endpoints}

The primary endpoint is the presence of parastomal hernia as detected by clinical examination or CT scan at 12 months after surgery. A bulge in the vicinity of the stoma is not sufficient in this study to define a parastomal hernia. If there is any doubt in the clinical setting, the patients must be examined by a CT scan in a prone position. Secondary endpoints include: re-admission rate, postoperative complications (classified according to Clavien-Dindo [27]), stoma-related complications (registered in the case record form (CRF) at Stoma Care nurse follow-up), total length of hospital stay during 12 months, health-related quality of life and health economic analysis, re-operation rate, mortality within 30 days and 12 months of primary surgery. Re-admissions and reoperations as registered in the hospitals' databases at 24 months to ascertain that possible long-term complications related to the stoma construction are identified.

\section{Inclusion criteria}

Patients are eligible if the following conditions are met:

- the patient is presenting with a condition for which an elective surgical procedure is planned including formation of a colostomy

- it is possible to operate on the patient in regard to concomitant disease

- the patient has given informed consent to participate

\section{Exclusion criteria}

- Participation in other randomised trials in conflict with the protocol and endpoints of the Stoma-Const trial.

\section{External validity}

All patients with a condition for which an elective surgical procedure is planned including a colostomy formation, and who are not included and randomised, will be registered in the 'screening log' at each participating centre including information on date, hospital, gender, age, ASA classification and type of operation. The reason for noninclusion or exclusion is noted.

\section{Randomisation}

All participating patients give written informed consent. After inclusion no changes are made in the planning for the operation. The patient will be randomised to one of the three groups of intervention during surgery in the operating theatre directly prior to the stoma formation (Figure 1). Randomisation will be performed by blocks of closed envelope systems in each participating hospital. It will be balanced and stratified by hospital. Laparoscopic as well as open procedures will be included and, although not stratified by open or laparoscopic procedures, their use will be noted in the CRF.

Including hospitals may choose to randomise between all three techniques or two of them. This decision is made before the hospital enters the study. The blocks will be set up according to this system, and the total number of 80 


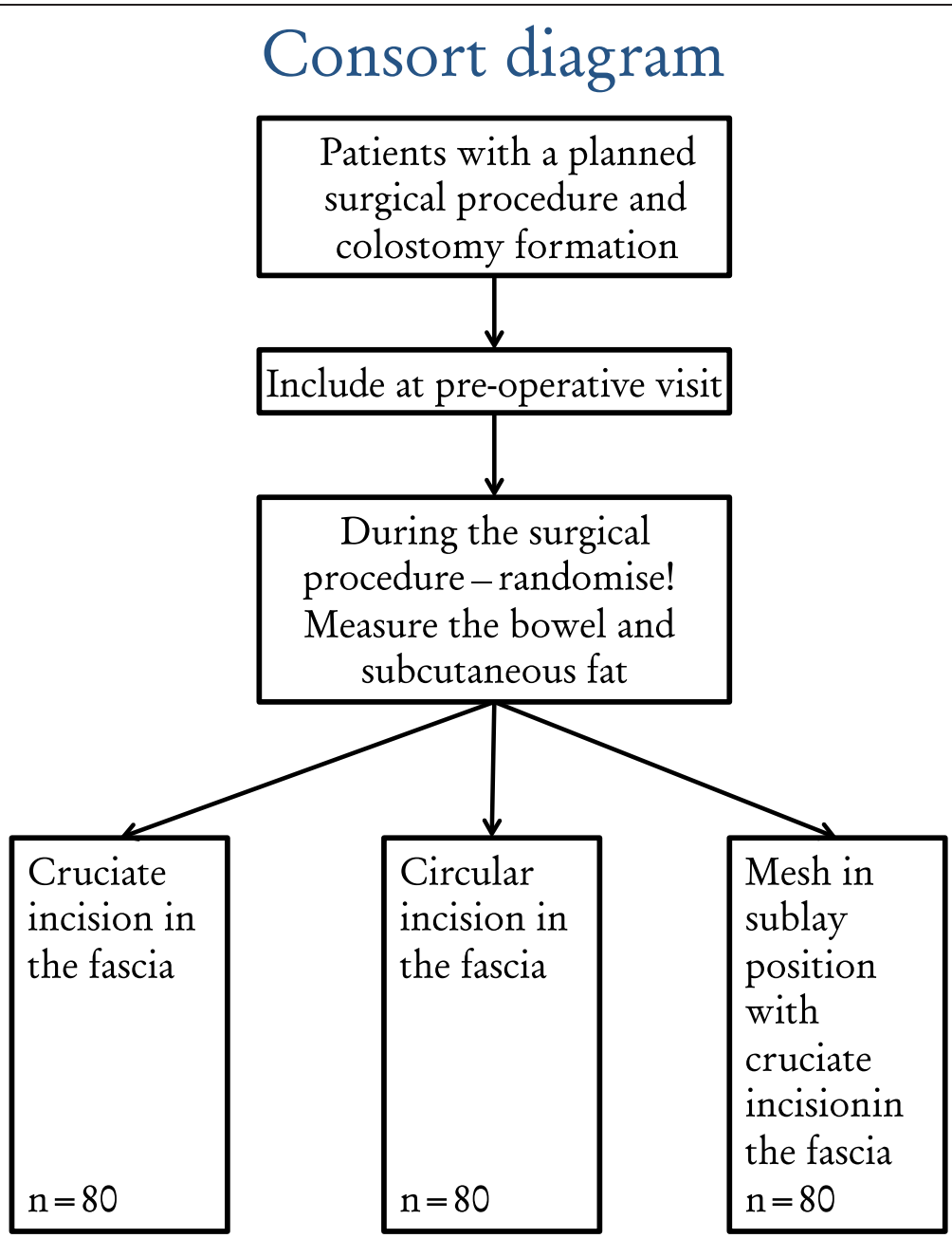

Figure 1 Consort diagram for the Stoma-Const trial.

patients in each group will be managed through this system.

When patients are not subject to the treatment modality as randomised, data will be analysed on an intention-totreat basis' (once randomised, patients will not be excluded or change groups because of conversion or type of surgery). Patients who do not consent to participation or are excluded will be treated by cross incision in the fascia as the usual routine.

\section{Surgical procedure}

A stoma care nurse marks all stoma sites preoperatively. At the time of the stoma formation, randomisation to one of the three (or in applicable cases two) procedures is made. The centre point of the preoperatively marked place for the stoma is lifted and a circular skin incision is performed. Blunt or 'semi-blunt' dissection is used through the subcutaneous tissue, using a step-wise downwards movement with retractors assisted by sharp dissection as needed. When the ventral fascia of the rectus abdominis has been reached, dissection is stopped. A disposable, sterile measurement tape is used in the open surgical procedures, and the metric scale on the laparoscopic stapler is used in laparoscopic surgical procedures. The depth of the subcutaneous fat is measured. The width of the left colon together with its mesocolon is measured at the point where it will pass through the fascia (the depth of the subcutaneous fat $+20-30 \mathrm{~mm}$ ). The fascia incision is calculated using this measurement - circular incision in the fascia with a diameter equalling $50 \%$ of the width of the patient's left colon with mesocolon, or a cruciate incision where the arms measure one-half of the width of the patient's left colon with mesocolon. If it is apparent that the incision will be too large, it may be reduced, but measured thoroughly and noted in the operative CRF. The muscular fibres are separated bluntly.

\section{Stoma formation without a mesh}

The dorsal fascia is opened. The proximal colon end is taken through the stoma incision (plica epiploicae may 
be removed, if necessary). Care is taken to ensure adequate circulation to the colon involved in the stoma. If the width of the incision is insufficient for adequate circulation, a widening should be performed, measured and noted in the CRF. The muco-cutaneous suturing is made using 4-0 absorbable interrupted sutures, three-point sutures (mucosa, muscular layer and skin) starting with one in each quadrant and adding ordinary muco-cutaneous sutures in between as needed. The height of the stoma after it is created should be at least $1 \mathrm{~cm}$.

\section{Stoma formation with a mesh}

The incision in the fascia is performed with a cruciate incision, with size according to the measurements described above, and the mesh will be placed using the sublay technique as previously described [14]. A finethread, large-pore lightweight polypropylene monofilament mesh measuring $10 \times 10 \mathrm{~cm}$ is used. The mesh is placed dorsal to the rectus abdominis muscle and anterior to the posterior rectus fascia by dissection through either midline in open surgery or by blunt dissection under the rectus abdominis muscle in laparoscopic surgery. A cruciate incision with a size measured according to the instructions above is made in the anterior sheath of the fascia and in the mesh. The bowel is brought out through a cross-cut in the centre of the mesh where the arms should measure one-half of the width of the patient's left colon and its mesocolon. If the width of the incision is not sufficient, a widening should be performed, measured and noted in the CRF. Stitches fix the lateral corners of the mesh to the posterior rectus sheath. The medial corners of the mesh are grasped with a stitch of the running suture closing the midline incision during open surgery, and this is not applicable to laparoscopic procedures.

The diameter of the stoma is measured immediately after the operation.

\section{Postoperative treatment}

Local guidelines are followed and must be applied equally in all three (in applicable cases, two) groups, without any systematic differences. Any postoperative treatment with antibiotics must be noted in the CRF, including reasons and total treatment time. This applies to both systemic as well as oral antibiotics. All postoperative information regarding stoma and stoma function will be given according to local routines.

\section{Follow-up}

The follow-up during the postoperative hospital stay is recorded in a CRF. Time until full oral feeding is resumed and time until first gas and stool are registered.

Patients will be clinically examined at 6 (5-7) months and at $12(11-13)$ months postoperatively and further as needed (suspected parastomal herniation), by a surgeon (not one of the colorectal team) preferably specialised in hernia surgery, to diagnose a possible parastomal hernia. At 12 months patients for whom no clinical parastomal herniation has been found will undergo a CT examination of the abdomen (wall), performed in prone position [25], which also will be part of the routine 12 months follow-up by abdomen CT for colorectal cancer patients. A study-specific standardised referral to abdomen CT is used, and although the study is not blinded, no information as to type of stoma construction is included. All radiologic departments use a specific protocol for this part of the abdomen CT.

Stoma height, diameter, colour, skin irritation and bandaging problems are documented by nurses specialised in stoma care during hospitalisation and at 4-6 weeks, 6 months and 12 months and are registered in a CRF.

\section{Quality of life}

All patients are asked to fill out questionnaires preoperatively, at 6 months and at 12 months after discharge from the hospital. The questionnaire has been constructed using previously used questions [28] that have been developed through a process of in-depth qualitative interviews and content validated by an expert panel consisting of colorectal surgeons, stoma care nurses and nurses specialised in surgery. The questionnaire was face-to-face validated by patients with a surgical procedure including a colostomy as well as patients with an existing colostomy using the validation methods described previously for a questionnaire for prostate cancer patients (26).

\section{Health economic evaluation}

A health economic analysis will be performed based on the information collected in the CRF together with information from the hospital registries, based on the model presented by Bjöholt et al. [29]. The model will be used in combination with sensitivity calculations.

\section{Data collecting and monitoring}

An operative CRF including reasons for surgery, planned operation and patient data, together with measurements from the surgical procedure is filled in for the operation (by the surgeon). For the hospitalisation period a nurse at the ward will complete a CRF, and for each follow-up visit a surgeon and a nurse will complete a CRF.

The postoperative questionnaires will be given to the patients by the stoma therapist. All data will be kept within University of Gothenburg and Sahlgrenska University Hospital systems using inherent security systems. A logistic database with complete patient ID will be used and kept within a separate IT system from the result database with all study information. Security measures will include one to maximum two users of this database, with unique usernames and personal login, as well as automatic 
throw-out. The questionnaires filled out by the patients are returned to the trial coordinating centre, SSORG, at Sahlgrenska University Hospital. The finalised database based on trial number and without patient ID, will be kept within another IT system than the logistic database.

\section{Statistical analysis}

The Swedish trial on the effect of prophylactic net placement has reported 50\% incidence of parastomal hernia without mesh [14]. Our own data on abdominoperineal excision from Sahlgrenska University Hospital indicate an incidence of $25 \%$ [28]. The power calculation based on available information has used the hypothesis that the cruciate incisional stoma formation, performed as described here, should result in parastomal hernia incidence of $30 \%$ and the circular incision or prophylactic mesh reinforcement could reduce this incidence to $10 \%$ each. An $80 \%$ power and a $5 \%$ level of significance with a two-sided alternative hypothesis would require 62 patients per group using a chi-square test. Expecting 20\% drop-outs, 80 patients will be recruited in each group.

The primary analysis will be based on the full analysis set. This analysis set is designed to be consistent with recommendations set forth in International Conference on Harmonisation ( $\mathrm{ICH})$ guidance document E9: Statistical Principles for Clinical Trials, and is intended to adhere as closely as practically possible to the intention-to-treat (ITT) principle. The full analysis set will consist of all randomised patients who had surgery and who contributed with any follow-up data.

Patients will be analysed according to randomised procedure. A sensitivity analysis evaluating the actual procedure will be made for the most important outcomes.

All hypothesis testing will be conducted using two-sided tests, and $\mathrm{P}$ values $\leq 0.05$ after rounding will be considered statistically significant. A pair-wise comparison between the experimental arms and the control arm will be performed and the significance level will be corrected using the Bonferroni-Holm method. Event-type variables (yes or no) will be compared between randomised procedures using chi-square tests, and relative risks and risk differences will be estimated. Where appropriate, the time to event will be described using Kaplan-Meier plots. The number of events of a certain type (for example, number of complications or re-operations) will be analysed using Poisson regression with randomised procedure as a factor and time under observation as an offset variable.

Total length of hospital stay will be compared between groups using Wilcoxon's rank-sum test. Sub-group analyses specifically looking at the following groups of patients will be performed:

a) $\mathrm{BMI}>30 \mathrm{~kg} / \mathrm{m} 2$

b) Immunosuppression
Sensitivity analyses will be performed for the primary variable and selected secondary variables, as deemed appropriate, where groups are compared pair-wise utilising only data from centres where both treatments were allocated.

Exploratory analyses (such as logistic regression) may be performed to investigate the influence of background factors (patient characteristics, perioperative factors) on outcomes.

\section{Participating hospitals}

The study will recruit patients from hospitals in Denmark and Sweden.

\section{Approvals and registration}

The trial has been approved by the Swedish Ethical Committee (EPN/Göteborg Dnr 547-12) and the Swedish radiotherapy protection committee (Dnr 12-38). It has been approved by the Danish ethics committee (Protocol H-4-2013-061), and by the Danish Data Protection Agency (no. HEH-2013-049, I-Suite no:02418). The study was registered at www.clinicaltrials.gov (NCT NCT01694238) prior to inclusion of the first patient.

\section{Discussion}

Parastomal hernia is a common complication after stoma creation $(12,13)$. There are several randomised trials that have tried to evaluate a reduction of the occurence of parastomal hernia with placement of a prophylactic mesh $[13,14,30]$. However, the use of a prophylactic measure must be evaluated in relation to health-related QoL as well as health economy. It is important to have enough power to thoroughly evaluate possible complications and side effects. A recent health technology assessment at Sahlgrenska University Hospital concluded that mesh probably is beneficial in regard to parastomal hernia formation, but that further studies are required [31]. At present there are at least six other randomised trials investigating the importance of a mesh for prevention of parastomal hernia, including one Norweigan study [32] with 60 patients, one Danish study that was stopped before including the planned 198 patients [33] and one Swedish study with 300 patients [34]. Sixty patients are randomised to mesh/no mesh in a Spanish study [35], another Spanish study is randomising 32 patients to intraperitoneal mesh placement [36] and 200 patients are randomised in a French study [37]. In addition there is a Dutch study, with 150 patients [38].

Our study will provide additional information complementing these studies through the evaluation of the surgical technique without a mesh in the control arm and in the interventional arms. Furthermore, the QoL and health economic evaluation will enable improved decisionmaking regarding prophylactic measures against parastomal herniation. 


\section{Trial status}

The trial is ongoing and recruiting patients at centres in both Denmark and Sweden. Hospitals interested in participating are welcome to contact the corresponding author.

\author{
Abbreviations \\ ASA classification: American Society of Anaesthesiology classification; \\ BMI: body mass index; CRF: case record form; CT: computed tomography; \\ EPN: Ethical committee; ICH: International Conference on Harmonisation; \\ ID: identification; IT: information technology; ITT: intention to treat; \\ QoL: quality of life.
}

\section{Competing interests}

The authors declare that they have no competing interests.

\section{Authors' contributions}

ACM participated in the design of the study and the study protocol, CRFs and questionnaires, and the statistical considerations as well as in drafting of the manuscript. SE participated in the preparation of the study protocol, CRFs and questionnaires and drafting of the manuscript. EH conceived the study, participated in the design of the study and the study protocol, CRFs and questionnaires and the statistical considerations as well as in drafting of the manuscript. JE performed the statistical analysis plan and the drafting of the manuscript. UA participated in the design of the study and the study protocol as well as drafting of the manuscript. JR participated in the design of the study and the study protocol as well as drafting of the manuscript. FH participated in the design of the study and the study protocol as well as drafting of the manuscript. EA participated in the design of the study and the study protocol, CRFs and questionnaires and the statistical considerations and performed the drafting of the manuscript. All authors read and approved the final manuscript.

\section{Acknowledgements}

The authors are grateful for assistance in the design phase of the study provided by the stoma care nurses at Sahlgrenska University Hospital/Östra, especially Eva Carlsson and Charlotta Petersén.

\section{Funding}

The Swedish Cancer Society number CAN2013/500, Sahlgrenska University Hospital (ALF), 'Agreement concerning research and education of doctors' AFGBF 366481, The Health and Medical Care Committee of the Regional Executive Board, Region Västra Götaland VGRFOU384331 and VGRFOU 308311 all contributed to this trial.

\section{Author details}

${ }^{1}$ Department of Surgery, Institute of Clinical Sciences, SSORG - Scandinavian Surgical Outcomes Research Group, University of Gothenburg, Sahlgrenska University Hospital/Östra, 41685 Gothenburg, Sweden. ${ }^{2}$ Department of Anaesthesia, Surgery and Intensive Care, Sahlgrenska University Hospital/ Östra, SE-416 85 Gothenburg, Sweden. ${ }^{3}$ Department of Surgery, Herlev University Hospital, Copenhagen, Denmark. ${ }^{4}$ Department of Surgical Gastroenterology, Rigshospitalet, Copenhagen, Denmark.

Received: 13 January 2014 Accepted: 12 June 2014

Published: 27 June 2014

\section{References}

1. Pachler J, Wille-Jorgensen P: Quality of life after rectal resection for cancer, with or without permanent colostomy. Cochrane Database Syst Rev 2012, 12, CD004323.

2. Shabbir J, Britton DC: Stoma complications: a literature overview. Colorectal Dis 2010, 12:958-964.

3. Porter JA, Salvati EP, Rubin RJ, Eisenstat TE: Complications of colostomies. Dis Colon Rectum 1989, 32:299-303.

4. Cottam J, Richards K, Hasted A, Blackman A: Results of a nationwide prospective audit of stoma complications within 3 weeks of surgery. Colorectal Dis 2007, 9:834-838.
5. Persson E, Berndtsson I, Carlsson E, Hallen AM, Lindholm E: Stoma-related complications and stoma size - a 2-year follow up. Colorectal Dis 2010, 12:971-976

6. Parmar K, Zammit M, Smith A, Kenyon D, Lees N: A prospective audit of early stoma complications in colorectal cancer treatment throughout the Greater Manchester and Cheshire colorectal cancer network. Colorectal Dis 2011, 13:935-938.

7. Nguyen $\mathrm{MH}$, Pittas F: How large should a skin trephine be for an end stoma? Aust N Z J Surg 1999, 69:675-676.

8. Keighley MR, Williams NS: Surgery of the Anus, Rectum and Colon Philadelphia, PA: Saunders Elsevier; 2008.

9. Carne PW, Robertson GM, Frizelle FA: Parastomal hernia. Br J Surg 2003, 90:784-793.

10. Pilgrim $\mathrm{CH}$, Mclntyre R, Bailey M: Prospective audit of parastomal hernia: prevalence and associated comorbidities. Dis Colon Rectum 2010, 53:71-76.

11. Sjodahl $R$, Anderberg $B$, Bolin T: Parastomal hernia in relation to site of the abdominal stoma. Br J Surg 1988, 75:339-341.

12. Lian L, Wu XR, He XS, Zou YF, Wu XJ, Lan P, Wang JP: Extraperitoneal vs. intraperitoneal route for permanent colostomy: a meta-analysis of 1,071 patients. Int J Colorectal Dis 2012, 27:59-64.

13. Serra-Aracil X, Bombardo-Junca J, Moreno-Matias J, Darnell A, Mora-Lopez L, Alcantara-Moral M, Ayguavives-Garnica I, Navarro-Soto S: Randomized, controlled, prospective trial of the use of a mesh to prevent parastomal hernia. Ann Surg 2009, 249:583-587.

14. Janes A, Cengiz Y, Israelsson LA: Preventing parastomal hernia with a prosthetic mesh. Arch Surg 2004, 139:1356-1358.

15. Janes A, Cengiz Y, Israelsson LA: Preventing parastomal hernia with a prosthetic mesh: a 5-year follow-up of a randomized study. World J Surg 2009, 33:118-121. discussion 122-113.

16. Tam KW, Wei PL, Kuo LJ, Wu CH: Systematic review of the use of a mesh to prevent parastomal hernia. World J Surg 2010, 34:2723-2729.

17. Shabbir J, Chaudhary BN, Dawson R: A systematic review on the use of prophylactic mesh during primary stoma formation to prevent parastomal hernia formation. Colorectal Dis 2012, 14:931-936.

18. Janes $A$, Cengiz $Y$, Israelsson LA: Experiences with a prophylactic mesh in 93 consecutive ostomies. World J Surg 2010, 34:1637-1640.

19. Evans MD, Williams GL, Stephenson BM: Preventing parastomal herniation: is prophylactic prosthetic mesh absolutely necessary? World J Surg 2009, 33:1538-1539. author reply 1540-1531.

20. Wang J, Dou Z, Wang T, Liu D, Wang L: Circular stapler-assisted extraperitoneal colostomy. Dig Surg 2010, 27:521-524.

21. Resnick S: New method of bowel stoma formation. Am J Surg 1986, 152:545-548.

22. Wijeyekoon SP, Gurusamy K, El-Gendy K, Chan CL: Prevention of parastomal herniation with biologic/composite prosthetic mesh: a systematic review and meta-analysis of randomized controlled trials. J Am Coll Surg 2010, 211:637-645.

23. Sajid MS, Kalra L, Hutson K, Sains P: Parastomal hernia as a consequence of colorectal cancer resections can prophylactically be controlled by mesh insertion at the time of primary surgery: a literature based systematic review of published trials. Minerva Chir 2012, 67:289-296.

24. Helgstrand F, Gogenur I, Rosenberg J: Prevention of parastomal hernia by the placement of a mesh at the primary operation. Hernia 2008, 12:577-582.

25. Janes A, Weisby L, Israelsson LA: Parastomal hernia: clinical and radiological definitions. Hernia 2011, 15:189-192.

26. Gurmu A, Gunnarsson U, Strigard K: Imaging of parastomal hernia using three-dimensional intrastomal ultrasonography. Br J Surg 2011, 98:1026-1029.

27. Clavien PA, Barkun J, de Oliveira ML, Vauthey JN, Dindo D, Schulick RD, de Santibanes E, Pekolj J, Slankamenac K, Bassi C, Graf R, Vonlanthen R, Padbury $\mathrm{R}$, Cameron JL, Makuuchi M: The Clavien-Dindo classification of surgical complications: five-year experience. Ann Surg 2009, 250:187-196.

28. Angenete E, Correa-Marinez A, Heath J, Gonzalez E, Wedin A, Prytz M, Asplund D, Haglind E: Ostomy function after abdominoperineal resection - a clinical and patient evaluation. Int J Colorectal Dis 2012, 27:1267-1274.

29. Bjoholt I, Janson M, Jonsson B, Haglind E: Principles for the design of the economic evaluation of COLOR II: an international clinical trial in surgery comparing laparoscopic and open surgery in rectal cancer. Int J Technol Assess Health Care 2006, 22:130-135. 
30. Lopez-Cano M, Lozoya-Trujillo R, Quiroga S, Sanchez JL, Vallribera F, Marti M, Jimenez LM, Armengol-Carrasco M, Espin E: Use of a prosthetic mesh to prevent parastomal hernia during laparoscopic abdominoperineal resection: a randomized controlled trial. Hernia 2012, 16:661-667.

31. Correa Marinez A, Bengtsson J, Eriksson M, Hjalmarsson Y, Palmqvist E, Sjögren $P$, Jivegård L: End colostomy - with or without mesh reinforcement [Terminal kolostomi - med eller utan nätförstärkning]. Göteborg: Sahlgrenska Universitetssjukhuset HTA-centrum; 2013. Regional activity-based HTA 2013:62. Västra Götalandsregionen.

32. Peristomal Mesh for Prophylaxis of Parastomal Hernia. http://linicaltrials.gov/ show/NCT00496418.

33. Prevention of Parastomal Hernia by Primary Mesh Insertion. http://clinicaltrials. gov/show/NCT00641342.

34. Trial Concerning the Frequency of Parastomal Hernia With or Without a Mesh (STOMAMESH). http://clinicaltrials.gov/show/NCT00917995.

35. Role of Prosthetic Mesh in Preventing Parastomal Hernias (RPMPPH). http://clinicaltrials.gov/ct2/show/NCT01955278.

36. Prospective Study of the Use of a Mesh to Prevent Parastomal Hernia after Laparoscopic Abdominoperineal Resection. http://clinicaltrials.gov/ct2/show/ NCT01722565.

37. Primary Prevention of Peristomial Hernias via Parietal Prostheses. http://clinicaltrials.gov/ct2/show/NCT01380860.

38. Brandsma HT, Hansson BM, Haan HV, Aufenacker TJ, Rosman C, Bleichrodt RP: PREVENTion of a parastomal hernia with a prosthetic mesh in patients undergoing permanent end-colostomy; the PREVENT-trial: study protocol for a multicenter randomized controlled trial. Trials 2012, 13:226.

doi:10.1186/1745-6215-15-254

Cite this article as: Correa Marinez et al: Stoma-Const - the technical aspects of stoma construction: study protocol for a randomised controlled trial. Trials 2014 15:254.

\section{Submit your next manuscript to BioMed Central and take full advantage of:}

- Convenient online submission

- Thorough peer review

- No space constraints or color figure charges

- Immediate publication on acceptance

- Inclusion in PubMed, CAS, Scopus and Google Scholar

- Research which is freely available for redistribution 